\title{
Transcranial direct current stimulation - what is the evidence for its efficacy and safety? Abraham P Arul-Anandam ${ }^{1,2}$, Colleen Loo ${ }^{1,2 *}$ and Perminder Sachdev ${ }^{1,3}$
}

\author{
Addresses: ${ }^{1}$ School of Psychiatry, University of New South Wales, Sydney, Australia; ${ }^{2}$ Black Dog Institute, Prince of Wales Hospital, Hospital Road, \\ Randwick, NSW 2031, Australia; ${ }^{3}$ Neuropsychiatric Institute, Euroa Centre, Prince of Wales Hospital, Hospital Road, Randwick, NSW 2031, \\ Australia \\ * Corresponding author: Colleen Loo (colleen.loo@unsw.edu.au) \\ FI000 Medicine Reports 2009, I:58 (doi:I0.3410/MI-58)
}

The electronic version of this article is the complete one and can be found at: http://FI000.com/Reports/Medicine/content/I/58

\begin{abstract}
Transcranial direct current stimulation (tDCS), a non-invasive brain stimulation technique, has emerged in the past decade as a useful investigative and therapeutic technique. A number of recent studies suggest that tDCS is safe and may be efficacious in the treatment of a variety of psychiatric and neurological disorders, including major depressive disorder, chronic neuropathic pain, and stroke. More evidence is necessary, however, before it can be recommended for general clinical application.
\end{abstract}

\section{Introduction and context}

There is increasing interest in the therapeutic and investigative capabilities of non-invasive forms of brain stimulation, such as transcranial direct current stimulation (tDCS). tDCS is a non-invasive brain stimulation technique that applies a mild (1-2 mA) direct electrical current via the scalp to enhance or diminish neuronal excitability. There is strong evidence that neurons underlying the anode are 'excited', with resting membrane potential shifting towards depolarisation and an increased rate of spontaneous neuronal firing. By contrast, neurons underlying the cathode are 'inhibited', with resting membrane potential shifting towards hyperpolarisation and reduced neuronal firing $[1,2]$. This phenomenon has been utilised to investigate cortical function by either stimulating cortical regions with anodal stimulation or creating 'functional lesions' with cathodal stimulation, and then conducting neuropsychological testing. tDCS has also been investigated as potential treatment for neurological and psychiatric diseases, based on the principle of modulation of the excitability of stimulated cortical regions. Clinical trials using tDCS to treat major depressive disorder (MDD) in the 1960s and 1970s produced inconsistent findings, most likely due to variations in the stimulation techniques used (see [3-5] for reviews). By contrast, two recent randomised controlled trials [6,7] have suggested that tDCS has antidepressant effects and is safe. MDD is a common illness with an approximate lifetime prevalence of $17 \%$ and confers a large burden of disease in the community, so the prospect of effective non-invasive physical treatments is of considerable interest.

\section{Recent advances}

\section{Transcranial direct current stimulation as treatment}

Improved understanding of neuropathology and parameters of stimulation (especially electrode size and placement, stimulation strength and duration) has led to refined tDCS techniques in the past decade. Using these newer techniques, two double-blinded, sham-controlled studies using left prefrontal tDCS ( 1 and $2 \mathrm{~mA}, 20$ minutes per day for up to 10 days) reported positive results in reducing depressive symptoms [6,7]. One trial reported 69\% improvement in mean Hamilton Depression Rating Scale (HDRS) scores after five sessions of active tDCS over 1.5 weeks, compared with $30 \%$ improvement in the sham group [6]. Another trial also reported positive findings, with improvement in the active group on HDRS of $40.5 \%$, compared to $10.4 \%$ in the sham group, after 10 consecutive weekdays of treatment [7]. Moreover, these differences in outcome 
between active and sham groups persisted at 1-month follow-up, and an open-label extension of the trial indicated that tDCS had similar efficacy, but more rapid onset, compared to a 6-week course of $20 \mathrm{mg} /$ day fluoxetine, a selective serotonin re-uptake inhibitor antidepressant [8].

A more recent, non-placebo controlled clinical trial involving hospitalised patients with drug-resistant depression at high risk of suicide, referred for electroconvulsive therapy, reported $>30 \%$ improvement in depression rating scores [HDRS and BDI (Beck Depression Inventory)] after anodal stimulation to the left dorsolateral prefrontal cortex (2 mA, twice-daily, for 5 days) [9]. As this was an uncontrolled study, it is uncertain if administering tDCS twice per day was more efficacious than once-daily treatment.

On the other hand, modern tDCS techniques have failed to replicate the dramatic findings of older clinical trials on mood in healthy subjects. In a double-blind crossover trial, 21 subjects received bilateral frontal tDCS with an extracephalic reference electrode [10]. No significant effects on emotional state, affect, emotional decisionmaking, arousal and psychomotor function were found.

A recent sham-controlled trial with anodal stimulation to the primary motor cortex $(2 \mathrm{~mA}, 20$ minutes, five sessions) has also demonstrated the efficacy of tDCS for the treatment of chronic pain due to spinal cord injury [11]. Interestingly, the analgesic effect appeared to be cumulative, with greatest overall pain reduction after five sessions of treatment. Similar to the depression trial, differences in pain scores between the active and sham group were still present at 2-week follow-up. The same parameters have also been reported to alleviate pain in fibromyalgia [12].

There is also some evidence that tDCS may be effective in improving functional recovery after stroke. A randomised, double-blinded, sham-controlled trial found that cathodal tDCS (2 mA, 10 minutes) to the left frontotemporal region significantly improved performance on a picture-naming task in post-stroke patients with chronic aphasia immediately after stimulation [13].

An animal study in rats demonstrated that cathodal tDCS (100-200 $\mu \mathrm{A}, 15-60$ minutes) increased the threshold for localised seizure activity, suggesting a potential therapeutic role in epilepsy [14]. The clinical evidence for tDCS treatment of epilepsy in humans, however, remains limited (see [15] for a review).
Transcranial direct current stimulation as an investigatory tool

As a tool for investigating brain function, tDCS can help to clarify the causal links between neuroanatomy and behaviour, adding to evidence from other modalities such as neuroimaging. For example, changes in neuropsychological function during prefrontal tDCS imply that the stimulated areas are involved in the performance of that neuropsychological task. Anodal tDCS to the left prefrontal cortex (1 mA, 10 minutes) was shown to improve performance on a probabilistic classification learning task, which measures the unconscious retrieval of previous experiences [16]. Stimulation using the same parameters also reportedly enhanced working memory in healthy subjects, as measured by a three-back working memory task [17], and in patients with Parkinson's disease [18]. In these ways, tDCS has added to data that implicates the prefrontal cortex in both implicit learning and working memory. There is also some recent evidence that tDCS (1.5 mA, 15 minutes) to the temporoparietal areas in patients with Alzheimer's disease improves recognition memory performance [19].

\section{Mechanisms of action of transcranial direct current stimulation}

Recent neuroimaging studies have helped improve our understanding of the mechanisms of tDCS, and may help to further refine tDCS techniques in the future. Imaging modalities, including positron emission tomography [20], functional magnetic resonance imaging [21] and magnetic resonance spectroscopy [22,23] have suggested changes in regional blood flow, glutamatergic neurotransmission and membrane function after tDCS, including in brain regions distal to the site of stimulation.

In particular, it appears that tDCS is able to alter spontaneous neuronal firing rates without producing action potentials during stimulation. This is because the current densities produced by tDCS in the cortex are below the action potential threshold for cortical neurons $[24,25]$. Animal studies have shown that small voltage gradients from anodal and cathodal currents can respectively increase and decrease spontaneous neuronal firing $[1,2]$. In humans, tDCS-induced changes in motorevoked potential are attenuated by drugs that block sodium ion channels, suggesting that stimulation works, in part, by altering resting membrane potential $[26,27]$. There is also evidence that tDCS produces neuroplastic changes that enhance neurosynaptic transmission by modulating NMDA (N-methyl-D-aspartic acid) receptors (see [3] for a review). 


\section{Adverse effects}

A structural and diffusion-weighted magnetic resonance imaging study [28] compared the prefrontal cortex before and after anodal and cathodal tDCS, and found no structural alterations or disturbances of the bloodbrain barrier, and no reduction in apparent diffusion coefficient values (a marker of cytotoxic oedema). Other studies have reported no significant changes in levels of neuron-specific enolase (a sensitive marker of neuronal damage) immediately or 1 hour after tDCS [29]. Common side effects include mild headache, itching and erythema at the electrode site. There are no published reports of tDCS inducing seizure. For a comprehensive review of safety considerations, see [30].

\section{Implications for clinical practice}

The experimental findings described suggest that tDCS may emerge as a non-invasive therapeutic modality in the future, particularly for MDD, though there is a need for further replication in clinical trials, and clarification of the subgroup of patients most likely to benefit, before it can be recommended for clinical use. With a stronger evidence base, tDCS may present an attractive addition or alternative to available treatments for depression, particularly given its comparatively mild side-effect profile. For example, it may have a role in the treatment of patients unable to tolerate the side effects of antidepressant medications, and possibly in medication-refractory depression, if subsequent trials show efficacy in this subgroup. Moreover, tDCS has several advantages over other non-invasive forms of brain stimulation, such as transcranial magnetic stimulation: it is less expensive, less cumbersome and, therefore, more mobile, and may have longer lasting effects [31]. tDCS could thus be relatively easily implemented in a clinical setting. The evidence base is currently small, however, and more studies are needed before it can be recommended for general clinical application. In particular, a large multi-centric clinical trial is warranted to establish its efficacy and clinical utility. It is also too early to say whether optimal stimulation parameters have been discovered, and further work is necessary to establish this.

\section{Abbreviations \\ BDI, Beck Depression Inventory; HDRS, Hamilton Depression Rating Scale; NMDA, N-methyl-D-aspartic acid; MDD, major depressive disorder; tDCS, transcranial direct current stimulation.}

\section{Competing interests}

The authors declare that they have no competing interests.

\section{References}

I. Bindman LJ, Lippold OCJ, Redfearn JW: The action of brief polarizing currents on the cerebral cortex of the rat (I) during current flow and (2) in the production of long-lasting after-effects. J Physiol 1964, 172:369-82.

2. Purpura DP, McMurtry JG: Intracellular activities and evoked potential changes during polarization of the motor cortex. J Neurophysiol 1965, 28:166-85.

3. Arul-Anandam AP, Loo C: Transcranial direct current stimulation: A new tool for the treatment of depression? J Affect Disord 2009, [Epub ahead of print].

4. Nitsche, Michael A, Boggio PS, Fregni F, Pascual-Leone A: Treatment of depression with transcranial direct current stimulation (tDCS): a review. Exp Neurol 2009, [Epub ahead of print].

5. Murphy DN, Boggio P, Fregni F: Transcranial direct current stimulation as a therapeutic tool for the treatment of major depression. Curr Opin Psychiatry 2009, 22:306-II.

6. Fregni F, Boggio PS, Nitsche M, Marcolin MA, Rigonatti SP, PascualLeone A: Treatment of major depression with transcranial direct current stimulation. Bipolar Disord 2006, 8:203-4.

7. Boggio PS, Rigonatti SP, Ribeiro RB, Myczkowski ML, Nitsche MA, Pascual-Leone AP, Fregni F: A randomized, double-blind clinical trial on the efficacy of cortical direct current stimulation for the treatment of major depression. Int J Neuropsychopharmacol 2008, II:249-54.

8. Rigonatti SP, Boggio PS, Myczkowski ML, Otta E, Fiquer JT, Ribeiro RB, Nitsche MA, Pasxual-Leone A, Fregni F: Transcranial direct stimulation and fluoxetine for the treatment of depression. Eur Psychiatry 2008, 23:74-6.

9. Ferrucci R, Bortolomasi M, Vergari M, Tadini L, Salvoro B, Giacopuzzi M, Barbieri S, Priori A: Transcranial direct current stimulation in severe, drug-resistant major depression. J Affect Disord 2009, [Epub ahead of print].

10. Koenigs M, Ukueberuwa D, Campion P, Grafman J, Wassermann E: Bilateral frontal transcranial direct current stimulation: Failure to replicate classic findings in healthy subjects. Clin Neurophysiol 2009, I 20:80-4.

II. Fregni F, Boggio PS, Lima MC, Ferreira MJ, Wagner T, Rigonatti SP, Castro AW, Souza DR, Riberto M, Freedman SD, Nitsche MA, Pascual-Leone A: A sham-controlled, phase II trial of transcranial direct current stimulation for the treatment of central pain in traumatic spinal cord injury. Pain 2006, I22:197-209.

12. Fregni F, Gimenes R, Valle AC, Ferreira MJ, Rocha RR, Natalle L, Bravo R, Rigonatti SP, Freedman SD, Nitsche MA, Pascual-Leone A, Boggio PS: A randomized, sham-controlled, proof of principle study of transcranial direct current stimulation for the treatment of pain in fibromyalgia. Arthritis Rheum 2006, 54:3988-98.

13. Monti A, Cogiamanian F, Marceglia S, Ferrucci R, Mameli F, MrakicSposta S, Vergari M, Zago S, Priori A: Improved naming after transcranial direct current stimulation in aphasia. $J$ Neurol Neurosurg Psychiatry 2008, 79:45I-3.

14. Liebetanz DF, Monte-Silva KK, Oliveira MB, Amancio-dos-Santos A, Nitsche MA, Guedes RC: After-effects of transcranial direct current stimulation (tDCS) on cortical spreading depression. Neurosci Lett 2006, 398:85-90.

15. Nitsche MA, Paulus W: Noninvasive brain stimulation protocols in the treatment of epilepsy: current state and perspectives. Neurotherapeutics 2009, 6:244-50.

16. Kincses TZ, Antal A, Nitsche MA, Bartfai O, Paulus W: Facilitation of probabilistic classification learning by transcranial direct current stimulation of the prefrontal cortex in the human. Neuropsychologia 2004, 42: I 13-7.

17. Fregni F, Boggio PS, Nitsche MA, Bermpohl F, Antal A, Feredoes E, Marcolin MA, Rigonatti SP, Silva MTA, Paulus W, Pascual-Leone A: Anodal transcranial direct current stimulation of prefrontal 
cortex enhances working memory. Exp Brain Res 2005, 1 66:23-30.

FI000 Factor 3.0 Recommended

Evaluated by Mark Hallett 18 Aug 2005

18. Boggio PS, Ferrucci R, Rigonatti SP, Priscila C, Nitsche M, PascualLeone A, Fregni F: Effects of transcranial direct current stimulation on working memory in patients with Parkinson's disease. J Neurol Sci 2006, 249:31-8.

19. Ferrucci R, Mameli F, Guidi I, Mrakic-Sposta S, Vergari M, Marceglia S, Cogiamanian F, Barbieri S, Scarpini E, Priori A: Transcranial direct current stimulation as a therapeutic tool for the treatment of major depression. Neurology 2008, 71:493-8.

20. Lang N, Siebner HR, Ward NS, Lee L, Nitsche MA, Paulus W, Rothwell JC, Lemon RN, Frackowiak RS: How does transcranial DC stimulation of the primary motor cortex alter regional neuronal activity in the human brain? Eur J Neurosci 2005, 22:495-504.

21. Baudewig J, Nitsche MA, Paulus W, Frahm J: Regional modulation of BOLD MRI responses to human sensorimotor activation by transcranial direct current stimulation. Magn Reson Med 200I, 45:I96-20I.

22. Arul-Anandam AP, Rae C, Loo C, Pigot M, Mitchell PB, Sachdev PS: A magnetic resonance imaging study of time course changes after transcranial direct current stimulation in healthy control subjects [abstract]. Aust N Z J Psychiat 2008, 423:46.

23. Rango M, Cogiamanian F, Marceglia S, Barberis B, Arighi A, Biondetti $P$, Priori $A$ : Myoinositol content in the human brain is modified by transcranial direct current stimulation in a matter of minutes: a IH-MRS study. Magn Reson Med 2008, 60:782-9.

24. Wagner T, Fregni F, Fecteau S, Grodzinsky A, Zahn M, PascualLeone A: Transcranial direct current stimulation: A computer-based human model study. Neurolmage 2007, 35: I I I3-24.

25. Tehovnik Ej: Electrical stimulation of neural tissue to evoke behavioral responses. J Neurosci Methods 1996, 65:I-I7.

26. Liebetanz D, Nitsche MA, Tergau F, Paulus W: Pharmacological approach to the mechanisms of transcranial DC-stimulationinduced after-effects of human motor cortex excitability. Brain 2002, I 25:2238-47.

27. Nitsche MA, Fricke $K$, Henschke $U$, Schlitterlau A, Liebetanz D, Lang N, Henning S, Tergau F, Paulus W: Pharmacological modulation of cortical excitability shifts induced by transcranial direct current stimulation in humans. J Physiol 2003, 553:293-301.

28. Nitsche MA, Niehaus L, Hoffmann KT, Hengst S, Liebetanz D, Paulus W, Meyer BU: MRI study of human brain exposed to weak direct current stimulation of the frontal cortex. Clin Neurophysiol 2004, I I 5:2419-23.

29. Nitsche MA, Paulus W: Sustained excitability elevations induced by transcranial DC motor cortex stimulation in humans. Neurology 200I, 57:1899-90।.

30. Nitsche MA, Liebetanz D, Lang N, Antal A, Frithjof T, Paulus W: Safety criteria for transcranial direct current stimulation (tDCS) in humans. Clin Neurophysiol 2003, I | 4:2220-2.

31. Fregni F, Freedman S, Pascual-Leone A: Recent advances in the treatment of chronic pain with non-invasive brain stimulation techniques. Lancet Neurol 2007, 6:188-91. 